\title{
Transcriptomic analysis of Eruca vesicaria subs. sativa lines with contrasting tolerance to polyethylene glycol-simulated drought stress
}

Bang-Lian Huang ${ }^{1,2}$, Xuan Li ${ }^{1}$, Pei Liu', Lan Ma ${ }^{1}$, Wenhua Wu' ${ }^{1}$ Xuekun Zhang ${ }^{3}$, Zaiyun $\mathrm{Li}^{4}$ and Bangquan Huang ${ }^{1 *}$

\begin{abstract}
Background: Eruca vesicaria subsp. sativa is one of the Cruciferae species most tolerant to drought stress. In our previous study some extremely drought-tolerant/sensitive Eruca lines were obtained. However little is known about the mechanism for drought tolerance in Eruca.

Methods: In this study two E. vesicaria subs. sativa lines with contrasting drought tolerance were treated with liquid MS/PEG solution. Total RNA was isolated from 7-day old whole seedlings and then applied to Illumina sequencing platform for high-throughput transcriptional sequencing.

Results: KEGG pathway analysis indicated that differentially expressed genes (DEGs) involved in alpha-Linolenic acid metabolism, Tyrosine metabolism, Phenylalanine, Tyrosine and tryptophan biosynthesis, Galactose metabolism, Isoquinoline alkaloid biosynthesis, Tropane, Piperidine and pyridine alkaloid biosynthesis, Mineral absorption, were all up-regulated specifically in drought-tolerant (DT) Eruca line under drought stress, while DEGs involved in ribosome, ribosome biogenesis, Pyrimidine metabolism, RNA degradation, Glyoxylate and dicarboxylate metabolism, Aminoacyl-tRNA biosynthesis, Citrate cycle, Methane metabolism, Carbon fixation in photosynthetic organisms, were all down-regulated. 51 DEGs were found to be most significantly up-regulated ( $\log _{2}$ ratio $\geq 8$ ) specifically in the DT line under PEG treatment, including those for ethylene-responsive transcription factors, WRKY and bHLH transcription factors, calmodulin-binding transcription activator, cysteine-rich receptor-like protein kinase, mitogenactivated protein kinase kinase, WD repeat-containing protein, OPDA reductase, allene oxide cyclase, aquaporin, Oacyltransferase WSD1, C-5 sterol desaturase, sugar transporter ERD6-like 12, trehalose-phosphate phosphatase and galactinol synthase 4. Eight of these 51 DEGs wre enriched in 8 COG and 17 KEGG pathways.

Conclusions: DEGs that were found to be most significantly up-regulated specifically in the DT line under PEG treatment, up-regulation of DEGs involved in Arginine and proline metabolism, alpha-linolenic acid metabolism and down-regulation of carbon fixation and protein synthesis might be critical for the drought tolerance in Eruca. These results will be valuable for revealing mechanism of drought tolerance in Eruca and also for genetic engineering to improve drought tolerance in crops.
\end{abstract}

Keywords: Eruca vesicaria subs. Sativa, Genotypes, Drought tolerance, RNA-seq, Gene expression

\footnotetext{
* Correspondence: huangbangquan@163.com

${ }^{1}$ Hubei Collaborative Innovation Center for Green Transformation of

Bio-Resources, College of Life Science, Hubei University, Wuhan 430062,

China

Full list of author information is available at the end of the article
}

(c) The Author(s). 2019 Open Access This article is distributed under the terms of the Creative Commons Attribution 4.0 International License (http://creativecommons.org/licenses/by/4.0/), which permits unrestricted use, distribution, and reproduction in any medium, provided you give appropriate credit to the original author(s) and the source, provide a link to the Creative Commons license, and indicate if changes were made. The Creative Commons Public Domain Dedication waiver (http://creativecommons.org/publicdomain/zero/1.0/) applies to the data made available in this article, unless otherwise stated. 


\section{Background}

Drought is one of the most important environmental stresses in the world [1-3]. Previous studies indicated that plant responses to drought stress is quite complicated and exploring drought tolerance mechanism in plants is still a challenge [4-7]. Next generation sequencing (NGS) using Illumina HiSeq2000 covers the transcriptome many folds and allows quantification of the detected transcripts [8, 9]. By using next generation sequencing the gene expression is quantified, making it possible for quantitative comparisons [10]. Many researchers have used transcriptome sequencing to study the responses to drought stress in crops [10-16].

Biological and genetic diversity exists among and within plant species regarding drought tolerance, however many of these adaptive mechanisms are not completely understood. Genome-wide identification of drought-responsive transcriptions using genotypes with contrasting droughttolerance will help revealing interactions among metabolic pathways in response to drought stress [12]. Eruca vesicaria subsp. sativa is one of the Cruciferae species most tolerant to drought stress $[17,18]$. In our previous study some extremely drought-tolerant/sensitive Eruca lines were obtained [19]. In this study, Illumina sequencing technology was used to identify the differentially expressed genes (DEGs) and their biochemical pathways related to drought tolerance in drought tolerant (DT) versus droughtsusceptible (DS) Eruca genotypes. Expression level of some selected drought-responsive genes was validated by using quantitative RT-PCR. To our knowledge, this is the first report about the whole transcriptome analysis in $E$. vesicaria subsp. sativa. The results provide transcriptome data valuable for understanding drought tolerance mechanism in Eruca and the differentially expressed genes could be valuable for improving drought tolerance in crops.

\section{Results}

\section{Overview of Illumina RNA sequencing and de novo} assembly of $E$. vesicaria subs. sativa transcriptome In total 314,322,210 raw reads after sequencing produced $254,660,002$ clean reads including 430,166 unique genes with N50 value of $638 \mathrm{bp}$ and average length of $511.56 \mathrm{bp}$. The unigene length ranged from 201 to $16,517 \mathrm{bp}$ (Table 1).

The unigenes included 94,463 clusters and 335,703 singletons. Among the unigenes there were 308,310 (71.67\%) with length from 200 to $500 \mathrm{bp}, 76,255$ (17.73\%) with length from $500 \mathrm{bp}$ to $1000 \mathrm{bp}, 24,662$ (5.73\%) with length from $1000 \mathrm{bp}$ to $1500 \mathrm{bp}, 11,229$ (2.61\%) with length from $1500 \mathrm{bp}$ to $2000 \mathrm{bp}, 9709$ (2.26\%) with length longer than $2000 \mathrm{bp}$, and no unigenes shorter than $200 \mathrm{bp}$ (Table 2).

Based on $\mathrm{Nr}$ annotation and the E-value distribution, it was found that among the annotated unigenes 51,486 $(37.02 \%)$ shared very strong homology (E-value $<10^{-60}$ ), $31,700(22.80 \%)$ had strong homology $\left(10^{-60}<\right.$ Evalue $<$ $\left.10^{-30}\right)$ and 55, 904 (40.19\%) showed homology $\left(10^{-30}<\mathrm{E}-\right.$
Table 1 Illumina RNA-Seq reads and de novo assembly statistics of Eruca transcriptome

\begin{tabular}{ll}
\hline Total number of raw reads & $314,322,210$ \\
Total number of clean reads & $254,660,002$ \\
Mean length of reads (bp) & 90 \\
Number of total unigenes & 430,166 \\
Mean length of unigenes (bp) & 511.56 \\
Minimum unigene length (bp) & 201 \\
Maximum unigene length (bp) & 16,517 \\
N50 (bp) & 638 \\
Unigenes annotated to Nr & 139,090 \\
Unigenes annotated to KEGG & 40,463 \\
Unigenes annotated to COG & 105,052 \\
\hline
\end{tabular}

value $<10^{-5}$ ) (Fig. 1). Among the unigenes 83,392 had identity between $22.86-80 \%$ and 55,698 higher than $80 \%$.

Regarding the species distribution, 83,034 unigene (59.70\%) had similarity between $19.32-80 \%, 56,056$ unigene $(40.30 \%)$ had similarity higher than $80 \%$. Of the 139 , 090 unigenes annotated to $\mathrm{Nr}, 36,254$ (26.06\%) had matches to sequences from Brassica napus, followed by that from Brassica rapa (27,894, 20.05\%), Eutrema salsugineum (5366, 3.86\%), Hordeum vulgare (3578, 2.57\%), Arabidopsis thaliana (3391, 2.44\%), Physcomitrella patens (3289, 2.36\%), Camelina sativa (3249, 2.34\%) (Fig. 2).

\section{COG annotation and enrichment}

In total 105,052 unigenes were anotated to COG (Clusters of Orthologous Groups of proteins) database and assigned to 25 COG functional clusters (Fig. 3), among which the "general function prediction only" cluster comprised the highest number of unigenes (15,472, 14.73\%), followed by the cluster "translation, ribosomal structure and biogenesis" (14,062, 13.39\%) and "Posttranslational modification, protein turnover, chaperones" (10,244, 9.75\%). By contrast, only 110 unigenes were classified into "Cell motility", 98 unigenes classified into "Nuclear structure" and 3 unigenes classified into "Extracellular structures" (Fig. 3).

\section{GO anotation and enrichment}

GO (Gene ontology) with $\mathrm{Nr}$ annotation indicated that 4953 unigenes were classified into 69 groups that could be categorized into three main classifications: "biological process" (2859), "cellular component" (643) and "molecular function" (1451). In the "molecular function" class the largest number of unigenes (984) were involved in "catalytic activity"; in the "cellular component" class the largest number of unigenes (168) were involved in "cell part"; in the "biological process" class the largest number of unigenes (534) were involved in "metabolic process" (Additional file 1). 
Table 2 Length distribution of the Unigenes

\begin{tabular}{llllllll}
\hline Length $(\mathrm{nt})$ & Total & $<200$ & $200-500$ & $500-1000$ & $1000-1500$ & $1500-2000$ & $\geq 2000$ \\
\hline Number of Unigenes & 430,166 & 0 & 308,310 & 76,255 & 24,662 & 11,229 & 9709 \\
$\%$ & 100 & 0.00 & 71.67 & 17.73 & 5.73 & 2.61 & 2.26
\end{tabular}

\section{KEGG pathway annotation and unigene enrichment} In total 28,295 unigenes were annotated to 344 KEGG pathways (Additional file 2). The number of unigenes in different KEGG pathways ranged from 1 to 3245 . The map with the highest unigene representation was Ribosome (ko03010, 3245 unigenes, $11.47 \%$ ), followed by Carbon metabolism (ko01200, 1483 unigenes, 5.24\%), Biosynthesis of amino acids (ko01230, 1360 unigenes, 4.81\%), Protein processing in endoplasmic reticulum (ko04141, 1230 unigenes, 4.35\%).

\section{KEGG pathways and DEGs related to drought tolerance}

Difference in gene expression between drought-tolerant/ sensitive Eruca lines under MS/PEG treatment was analyzed by mapping back to the previous de novo assembling results using RSEM. As shown in Fig. 4 there were 5560, 4654, 9605, 8979 and 13,951 unigenes showing differential expression including both up- and down-regulated unigenes in DT vs DS, DT-MS vs DT-PEG, DS-MS vs DSPEG, DS-MS vs DT-MS, DS-PEG vs DT-PEG, respectively.

In the DS-MS vs DS-PEG group, 646 DEGs were enriched in 14 KEGG pathways (Q value < 0.05), i.e., 405 in Ribosome, 32 in Drug metabolism-cytochrome P450, 32 in Plant-pathogen interaction, 26 in Tyrosine metabolism, 40 in Phenylalanine metabolism, 37 in Plant hormone signal transduction, 32 in Metabolism of xenobiotics by cytochrome P450, 30 in Chemical carcinogenesis, 36 in Phenylpropanoid biosynthesis, 47 in Glutathione metabolism, 54 in Lysosome, 7 in D-Glutamine and D-glutamate metabolism, 3 in Biosynthesis of siderophore group nonribosomal peptides and 1 in Atrazine degradation (Additional file 3).

In the DT-MS vs DT-PEG group, 395 DEGs were enriched in 8 KEGG pathways (Q value <0.05), i.e., 257 in ribosome, 42 in Plant-pathogen interaction, 20 in Phenylalanine metabolism, 31 in Arginine and proline

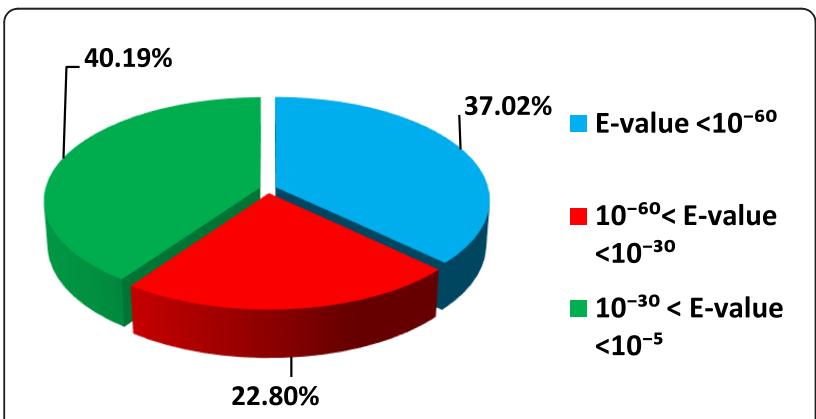

Fig. 1 Homology search of unigenes against the $\mathrm{Nr}$ database metabolism, 39 in Plant hormone signal transduction, 21 in Phenylpropanoid biosynthesis, 13 in alpha-Linolenic acid metabolism and 41 in D-Glutaminehe D-glutamate metabolism (Additional file 4).

Common pathways betweem DS-MS vs DS-PEG and DT-MS vs DT-PEG are Plant-pathogen interaction, Phenylalanine metabolism, Plant hormone signal transduction, Ribosome, Phenylpropanoid biosynthesis, D-Glutamine and D-glutamate metabolism; Pathways specifically in DS-MS vs DS-PEG are Drug metabolismcytochrome P450, Atrazine degradation, Tyrosine metabolism, Metabolism of xenobiotics by cytochrome P450, Biosynthesis of siderophore group nonribosomal peptides, Chemical carcinogenesis, Glutathione metabolismand Lysosome; pathways specifically in DT-MS vs DT-PEG are Arginine and proline metabolism and alpha-Linolenic acid metabolism (Additional files 3 and 4).

Referring to Audic's algorithm [20], FDR (False Discovery Rate) was calculated based on the $P$-value that corresponds to differential expression tests of transcripts. By using "FDR $\leq 0.05$ and the absolute value of $\log _{2}$ Ratio $\geq 1$ " as the threshold, we identified in the DS-MS vs DS-PEG group 3287 unigenes up-regulated and 6318 downregulated; in the DT-MS vs DT-PEG couple, 2299 unigenes up-regulated and 2355 down-regulated (Fig. 4). It was specifically in DT-MS vs DT-PEG but not in DS-MS vs DS-PEG that 2946 DEGs were detected, among which 1584 were up-regulated and 1362 down-regulated, 449 had $\mathrm{Nr}$ annotation and 552 annotated to 232 KEGG pathways (data not shown).

KEGG pathway analysis indicated that in DT-MS vs DT-PEG but not in DS-MS vs DS-PEG, DEGs involved in alpha-Linolenic acid metabolism, Tyrosine metabolism, Phenylalanine, tyrosine and tryptophan biosynthesis,

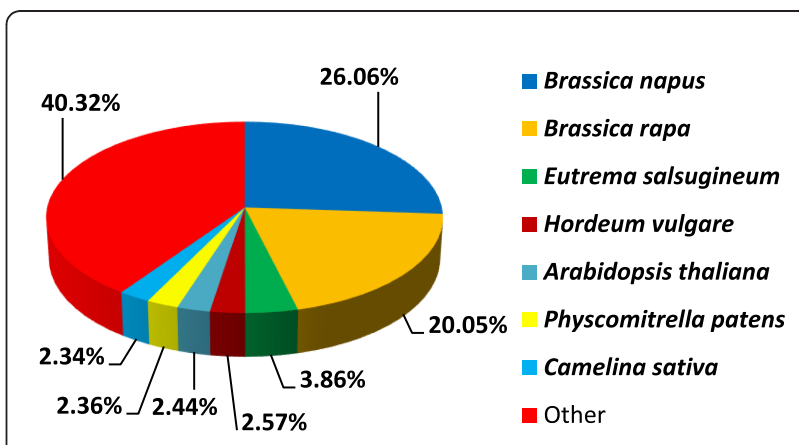

Fig. 2 Species distribution of Unigenes in the Nr database 


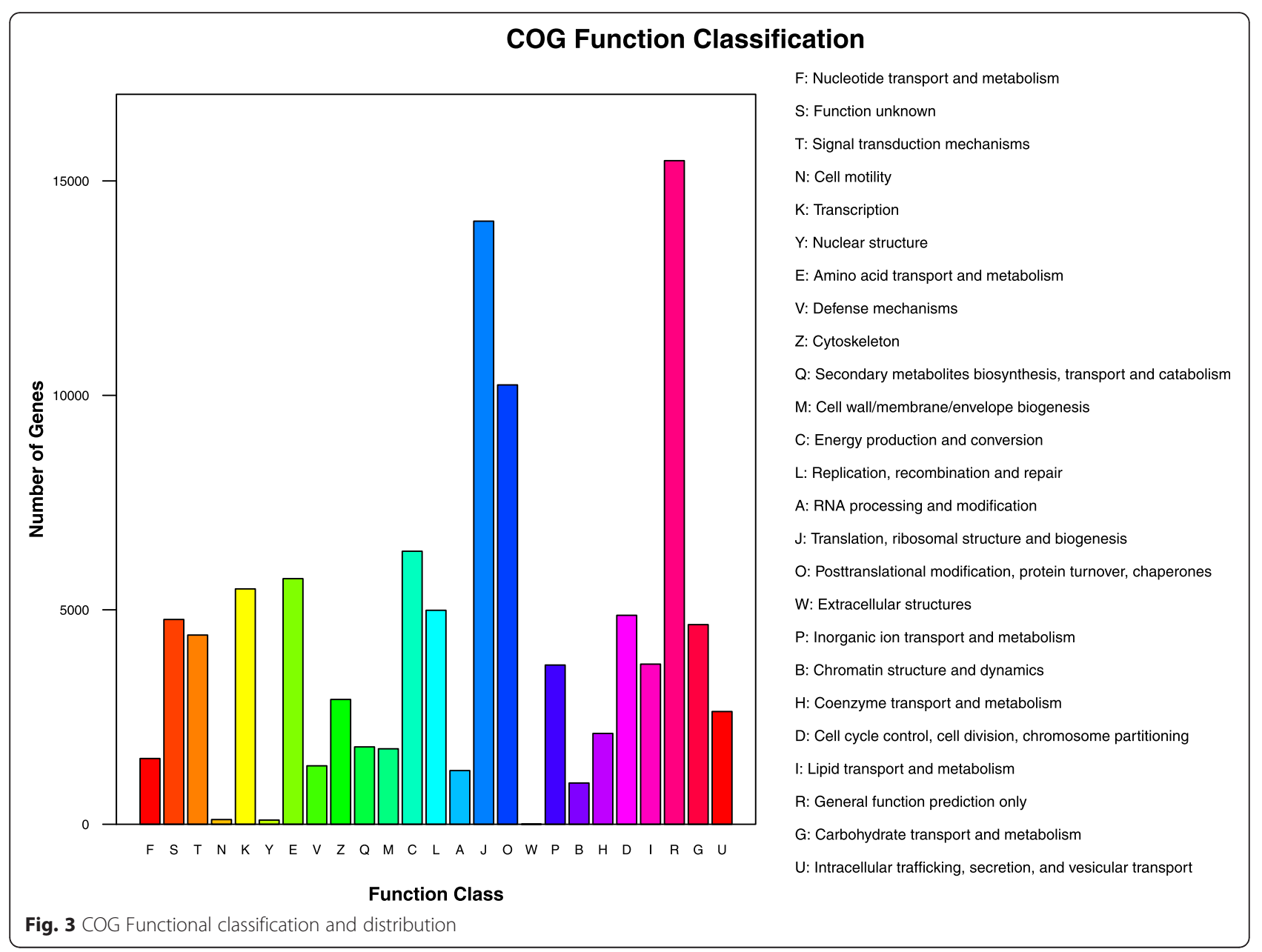

Galactose metabolism, Isoquinoline alkaloid biosynthesis, Tropane, piperidine and pyridine alkaloid biosynthesis, Mineral absorption, were all up-regulated $\left(\log _{2}\right.$ Ratio $\left.\geq 1\right)$; DEGs involved in ribosome, ribosome biogenesis, Pyrimidine metabolism, RNA degradation, Glyoxylate and dicarboxylate metabolism, Aminoacyl-tRNA biosynthesis, Citrate cycle, Methane metabolism, Carbon fixation in photosynthetic organisms, were all down-regulated $\left(\log _{2}\right.$ Ratio $\left.\leq-1\right)$. Most DEGs involved in Porphyrin and chlorophyll metabolism, Ubiquinone and other terpenoid-quinone biosynthesis, Arachidonic acid metabolism, Glutathione metabolism, glycerophospholipid metabolism, Phenylalanine metabolism,

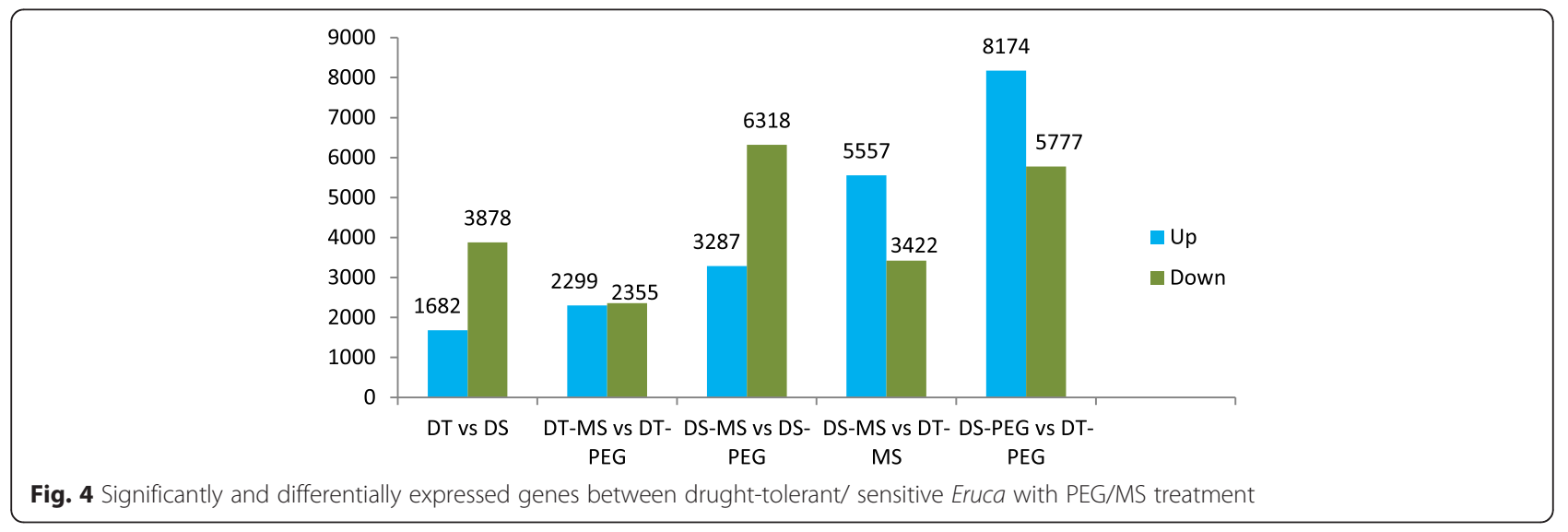


Plant-pathogen interaction, were up-regulated $\left(\log _{2}\right.$ Ratio $\geq$ 1); Most DEGs involved in Cell cycle, Peroxisome, Carbon metabolism, protein processing in ER, Regulation of actin cytoskeleton, Arginine and proline metabolism, Pyruvate metabolism, Pentose phosphate pathway, Nitrogen metabolism, Spliceosome, Apoptosis, Lysine degradation, lysosome, calcium signaling pathway, MAPK signaling pathway, Propanoate metabolism, Valine, leucine and isoleucine degradation, Alanine, aspartate and glutamate metabolism, Proteasome, Purine metabolism, RNA transport, Oxidative phosphorylation, down-regulated (Additional file 5).

Among the 2946 DEGs detected specifically in DT-MS vs DT-PEG but not in DS-MS vs DS-PEG, 1890 had blast hits against the NCBI database (Additional file 6), among which 51 DEGs were most significantly up-regulated $\left(\log _{2}\right.$ Ratio $\geq$ 8), including 3 DEGs for trehalose-phosphate phosphatase, 2 for allene oxide cyclase, 2 for ethylene-responsive transcription factor, 1 for aquaporin TIP1-1, 2 for sugar transporter ERD 6-like 12, 1 for transcription factor MYC3-like, 1 for senescence-associated carboxylesterase 101-like, 1 for calmodulin-binding transcription activator 2, 1 for cysteinerich receptor-like protein kinase 17,1 for galactinol synthase 4, 1 for mitogen-activated protein kinase kinase 4, 1 for transcription factor bHLH19-like, 1 for C5-sterol desaturase, 1 for WD repeat-containing protein, 1 for WRKY transcription factor, 1 for O-acyltransferase WSD1, 2 for low-temperature-induced $65 \mathrm{kDa}$ protein; 275 unigenes were most significantly down-regulated $\left(\log _{2}\right.$ Ratio $\leq-$ 8), including 38 DEGs for ribosomal protein. Fourteen of the 51 most significantly up-regulated DEGs were enriched in 8 COG pathways and 7 in 17 KEGG pathways (Additional file 7 and 8).

\section{RNA-seq validation: qRT-PCR analysis}

Quantitative RT-PCR (qRT-PCR) assays were carried out for 16 selected genes to verify the RNA-seq results. ERF4 (c189274 g1 i1), MLO2 (c205019 g2 i1), Ferritin (c194336 g1 i1), receptor-like protein kinase (c203474 g1 i1), dehydrin ERD14 (c194017 g1 i1), disease resistance protein RPP-13 (c207395 g1 i3), O-acyltransferase WSD1-like (c175262_g2_ i1), aquaporin TIP1 (c194457_g2_i2), pre-mRNA-splicing factor CLF1 (c161655_g2_i1), potassium transporter (c197830_g3_i1), senescence-associated carboxylesterase (c191638_g3_i8), transcription factor MYC3 (c187590_g3_ i3), EARLY FLOWERING 3 (c200550_g2_i1), were found to be up-regulated in DT-MS vs DT-PEG by qRT-PCR; proline dehydrogenase (c201592 g1 i1); NRT1 (c193030 g1 i3), ribosome (c178914 g1 i1), were found to be down-regulated in DT-MS vs DT-PEG by qRT-PCR. The qRT-PCR results are consistent with the RNA-seq results (Fig. 5 and 6).

\section{Discussion}

Comparative study using lines with contrasting droughttolerance is a useful tool for identifying drought-responsive genes. Identification of DEGs exclusively in the tolerant genotype would be valuable for revealing the mechanisms responsible for stress tolerance [21]. In the present study we provided detailed transcriptomic profiles of whole seedlings of two Eruca lines with contrasting drought tolerance.

\section{$A B A$ responses and stress signaling}

Drought stress induces ABA accumulation, which in turn leads to stomatal closure to keep the water status in plants [22-24]. Evidence indicates that WRKY proteins, including those induced by ABA, are upregulated under drought stress in rice [25]. OsWRKY45 overexpression increased drought tolerance [26], while activated expression of WRKY57 resulted in drought tolerance in Arabidopsis [27]. In this study 19 of 26 DEGs for WRKY transcription factor were significantly up-regulated specifically in the drought tolerant Eruca line under drought stress (hereafter referred as significantly upregulated), among which one for WRKY transcription factor 55-like (c195662 g1 i2) was most significantly upregulated specifically in drought-tolerant Eruca line PI 426649 under PEG-simulated drought stress (hereafter referred as most significantly up-regulated).

Basic helix-loop-helix (bHLH) genes are important in phytohormone signaling. Rice OsbHLH148 confers drought tolerance by interacting with OsJAZ proteins [28]. Arabidopsis bHLH122 and PebHLH35 from Populus euphratica are positive regulators of drought and salt tolerance and osmotic signaling $[29,30]$. In this study 5 DEGs for transcription factor bHLH were down-regulated and 8 up-regulated, among which one for transcription factor bHLH19-like (c196938 g3 i6) was most significantly up-regulated (Additional file 6).

\section{Ethylene-responsive transcription factors}

Previous studies have indicated that Ethylene-responsive transcription factors (ERFs) participate in abiotic stress responses in plants [31]. Transcription of GhERF4 was increased rapidly Gossypium hirsutum when plants were exposed to salt stress [32], and overexpression of ERF increased drought tolerance [33-36]. In this study 17 of the 18 DEGs for ethylene-responsive transcription factor were up-regulated, among which two for ERF056 (c163592 g1 i1, c163592 g2 i1) were most significantly up-regulated (Additional file 6).

\section{Antioxidants and ROS modulation}

Reactive oxygen species (ROS) often acts as secondary messenger modulating stomatal closure in responses to different stimuli [22, 37]. In Arabidopsis, calmodulinbinding transcription activator 1 (AtCAMTA1) is involved in regulation of membrane integrity by inducing ABA responses to drought stress. The camta1 mutants are highly susceptible to drought stress [38]. In this study 10 DEGs for calmodulin-binding protein were all 


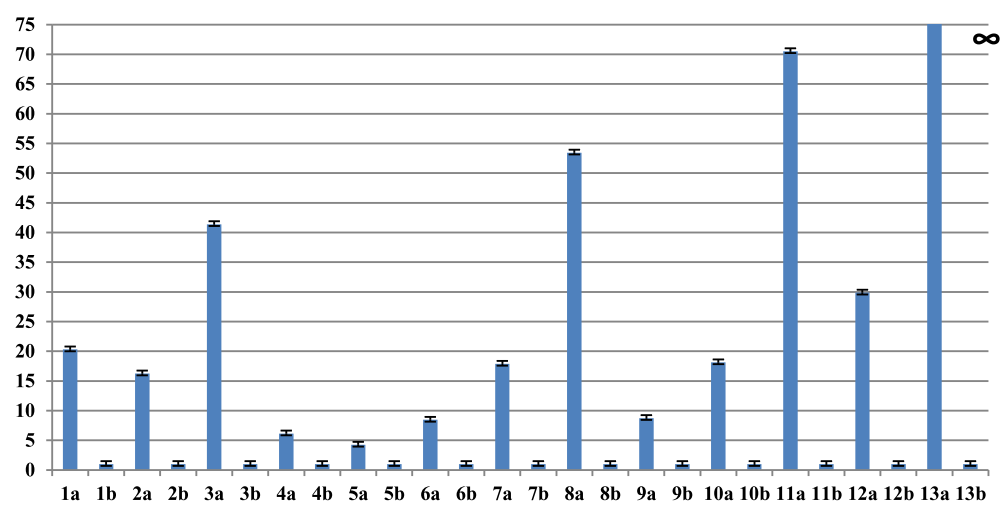

Fig. 5 Quantitative RT-PCR confirmation of some up-regulated Unigenes Axis Y represents the relative quantification (RQ) between the PEG and MS treated seedlings, while the error bars indicate standard deviation of three replicates. 1a:c189274_g1_i1-PEG; 1b:C189274_g1_i1-MS; 2a:c205019_g2_i1-PEG; 2b:c205019_g2_i1- MS; 3a:c194336_g1_i1-PEG; 3b:c194336_g1_i1-MS; 4a:c203474_g1_i1-PEG; 4b:c203474_g1_i1-MS; 5a:c194017_g1_i1; 5b:c194017_g1_i1; 6a:c207395_g1_i3-PEG; 6b:c207395_g1_i3-MS; 7a:c175262_g2_i1-PEG; 7b:c175262_g2_i1-MS; 8a:C194457_g2_i2-PEG; 8b:c194457_g2_i2-MS; 9a:C161655_g2_i1-PEG; 9b:c161655_g2_i1-MS; 10a:C197830_g3_i1-PEG; 10b:c197830_g3_i1-MS; 11a:c191638_g3_i8-PEG; 11 b:c191638_g3_i8-MS; 12a:c187590_g3_i3-PEG; 12b:c187590_g3_i3-MS; 13a:c200550_g2_i1-PEG; 13b:c200550_g2_i1-MS

up-regulated, among which one for calmodulin-binding transcription activator 2-like (c195596 g1 i7) was most significantly up-regulated (Additional file 6).

Mos Cysteine-rich receptor-like kinases (CRKs) are regulated by ROS [39]. It was found that CRK45 positively regulated plant responses to drought and salt stresses by inducing expression of ABA-responsive and stress-inducible genes. CRK45 overexpression enhanced drought tolerance in plants [40]. Overexpression of Arabidopsis CRK5 also increased ABA sensitivity and thus enhanced drought tolerance [41]. In this study 10 of 12 DEGs for cysteine-rich receptor-like protein kinase were up-regulated, among which one for cysteine-rich receptor-like protein kinase 17 (c196393 g1 i2) was most significantly up-regulated (Additional file 6).

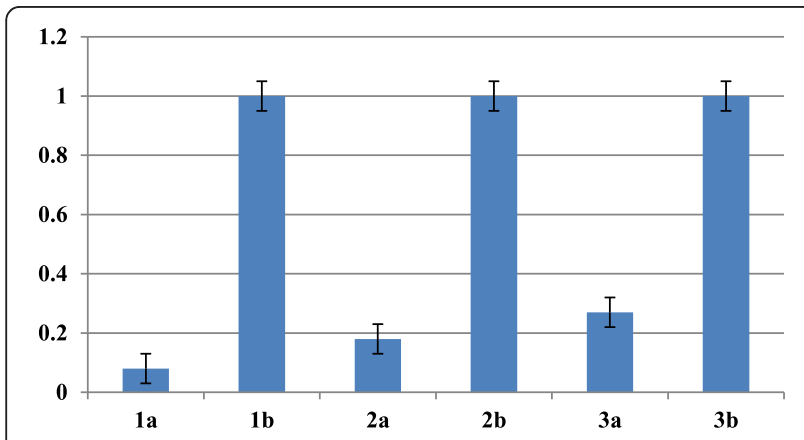

Fig. 6 Quantitative RT-PCR confirmation of some down-regulated Unigenes Axis $Y$ represents the relative quantification (RQ) between the PEG and MS treated Eruca seedlings, and the error bars indicate standard deviation of three replicates. 1a: proline dehydrogenase (c201592 g1 i1)-PEG; 1b: proline dehydrogenase (c201592 g1 i1)-MS; 2a: NRT1 (c193030 g1 i3)-PEG; 2b: NRT1 (c193030 g1 i3)- MS; 3a: ribosome (c178914 g1 i1)-PEG; 3b: ribosome (c178914 g1 i1) - MS
It has been shown that MAPKs are involved in plant signal transduction in responses to different environmental stimuli [42-46]. MAPK, MAPKK and MAPKK kinase constitute a functional MAPK cascade. Activation of MAPK helps its translocation to nucleus to phosphorylate and activate transcription factors [47]. AtMKK1 in Arabidopsis activates AtMPK3 to transfer abiotic stess signals [48]. NtMEK2 (MAPKK) activates WIPK and SIPK for drought signal transmission [49]. Overexpression of GhMKK1 increased drought tolerance in Nicotiana benthamiana [41]. Overexpression of AtMKK1 in Arabidopsis decreased ROS levels and increased drought tolerance, while AtMKK1 deficiency resulted in elevated ROS and increased sensitivity to drought tolerance [50]. Plants over expressing AtMKK4 accumulated fewer ROS and showed less water-loss under drought stress [51]). In this study one DEG for mitogen-activated protein kinase kinase 4 (c198487 g1 i1) was most significantly up-regulated (Additional file 6).

$N A C$ genes such as $S N A C 3$ contributes to drought resistance and osmotic modulation independent of ABA [52]. SNAC3 could interact with WD domain-containing protein to adjuste ROS in rice. In this study all 13 DEGs for NAC domain-containing protein were up-regulated and one DEG for WD repeat-containing protein 53 (c198319 g1 i2) was most significantly up-regulated (Additional file 6).

\section{Alpha-linolenic acid, jasmonate signaling and cell membrane stability}

Plants keep membrane fluidity and integrity by modulating oleic and linolenic acid levels during stresses [53]. Alphalinolenic acid is a major precursor for messengers including jasmonic acid generated by oxidative modifications [54-56]. Antisense expression of Arabidopsis omega-3 fatty acid 
desaturase gene led to reduced salt/drought tolerance in tobacco [57], while over-expression of FAD3 or FAD8 resulted in increased drought tolerance [58]. Lenka et al. [12] found that eight enzymes involved in alpha-linolenic acid metabolism were significantly induced by drought stress in drought-tolerant rice. In this study we also found all DEGs involved in alpha-linolenic acid metabolism up-regulated (Additional file 5).

Jasmonic acid (JA) plays critical role in stomatal closure during drought stress [59]. It is also involved in the production of antioxidants regulating ascorbate and glutathione metabolism [60]. Based on the pathway established by Vick and Zimmerman [61], JA is produced from alpha-linolenic acid, and in this process allene oxide cyclase and OPDA reductase (OPR) play important roles [62-66]. In this study all four DEGs of OPDA reductase and 6 DEGs for allene oxide cyclase were up-regulated, among which two DEGs for allene oxide cyclase (c184414 g1 i1, c184414 g2 i1) were most significantly up-regulated (Additional file 6).

Previous studies indicated that Aquaporins (AQPs) played an important role in decreasing ion leakage (IL) and malondialdehyde (MDA), thus reducing membrane injury caused by abiotic stresses [67]. Overexpression of banana Aquaporin gene MaPIP1;1 increased salt tolerance in Arabidopsis [68]. In this study one DEG for aquaporin TIP1-1 (c194457 g2 i2) was most significantly up-regulated (Additional file 6).

Cuticular waxes play important roles in reducing nonstomatal water loss under stresses [69]. C-5 sterol desaturase catalyzes the incorporation of $\mathrm{C}-5$ double bond into D7-sterols to form D5, 7-sterols. Overexpression of fungal C-5 sterol desaturase increased wax deposition and drought tolerance in tomato [70]. In this study one DEG for C-5 sterol desaturase (c201142 g1 i2) was most significantly up-regulated (Additional file 6).

O-acyltransferase WSD1 is also involved in cuticular wax biosynthesis [71]. Zhang et al. [35] indicated that $K C S$ and $W S D$, and their up-stream regulators, were upregulated under drought stress. In this study one DEG for O-acyltransferase WSD1-like (c175262 g2 i1) was most significantly up-regulated (Additional file 6).

\section{Trehalose, galactinol and sugar transport}

In plants various abiotic stresses lead to sugar accumulation in the vacuole [72-74], suggesting that sugar biosynthesis and vacuolar sugar transporters play important roles under these conditions. Sugar transporter ERD6 expression is induced by drought stress [75], while ERD6-like transporter (ESL1) expression is enhanced by drought and $\mathrm{ABA}$ treatment [76]. In this study 2 DEGs for sugar transporter ERD 6-like 12 (c197650_g1_i3, c197650_g1_i5) were most significantly up-regulated (Additional file 6).
Trehalose is also important in protecting plants from stresses [77]. Trehalose-6-phosphate (T6P) is suggested to act as signaling metabolite responsing to the environment [78]. In plants T6P is dephosphorylated by trehalose-6phosphate phosphatase to produce trehalose [79]. In this study all 6 DEGs for trehalose-phosphate phosphatase were up-regulated, among which 3 were most-upregulated (Additional file 6).

Galactinol synthase (GolS) plays important roles in carbon partitioning between sucrose and RFOs and the GolS gene products are reported to function in responses to abiotic stresses [80-83]. Transgenic Arabidopsis and Brachypodium distachyon overexpressing AtGolS2 accumulated more galactinol and raffinose and showed enhanced drought tolerance $[84,85]$. In this study one unigene for galactinol synthase 4 (c194151 g2 i3) was most significantly up-regulated (Additional file 6).

Down-regulation of carbon fixation and protein synthesis Lenka et al. [12] found that under drought stress carbon fixation was up-regulated, while in this study we found that in the drought-tolerant Eruca line DEGs for carbon fixation in photosynthetic organisms were all downregulated under drought stress (Additional file 5). Ribosomes are places where proteins are synthesized. Dhindsa and Bewley [86] found decline of amino acid incorporation and polysome population in the droughttolerant moss Tortula ruralis under drought stress. In this study KEGG pathway analysis indicated that DEGs involved in ribosome, ribosome biogenesis, AminoacyltRNA biosynthesis were all down-regulated in drought tolerant Eruca under PEG-simulated drought stress (Additional file 5); Blast analysis also indicated that 9 of 10 DEGs for translation initiation factor, 8 DEGs of ATP synthase, 6 DEGs for ribosomal RNA gene, 5 of 6 DEGs for eukaryotic translation initiation factor, 118 of 121 DEGs for ribosomal protein, were down regulated, among which 38 DEGs for ribosomal protein were most significantly down-regulated (Additional file 6), suggesting that Eruca might also respond to drought stress by down-regulation of protein synthesis and carbon fixation in photosynthetic organisms.

\section{Other pathways and DEGs possibly related to drought tolerance}

In this study it was found that two DEGs for lowtemperature-induced $65 \mathrm{kDa}$ protein-like (c183129 g1 i2, c183129 g3 i4), one for lipase ROG1 (c185493 g1 i1), one for disease resistance protein (c192485 g1 i11), one for amino-acid permease BAT1 (c205647 g1 i3), one for ankyrin repeat-containing protein (c201331 g1 i3), one for acyl-activating enzyme 19 (c188918 g1 i1), one for phospholipid hydroperoxide glutathione peroxidase 6 (c195141 g2 i3), one for potassium transporter 9-like 
(c197830 g3 i1), one for phosphoglycerate mutase-like protein 2 (c190346 g1 i4) were most significantly upregulated (Additional file 6). Their roles in drought tolerance need to be further explored.

KEGG pathways in which all DEGs were up-regulated, such as Tyrosine metabolism, Phenylalanine, tyrosine and tryptophan biosynthesis, Galactose metabolism, Isoquinoline alkaloid biosynthesis, Tropane, piperidine and pyridine alkaloid biosynthesis, Mineral absorption; KEGG pathways in which most DEGs were up-regulated, such as Porphyrin and chlorophyll metabolism, Ubiquinone and other terpenoid-quinone biosynthesis, Arachidonic acid metabolism, Glutathione metabolism, glycerophospholipid metabolism, Phenylalanine metabolism, Plant-pathogen interaction, were up-regulated (Additional file 5). Blast analyses indicated that all 5 DEGs for dehydration-responsive elementbinding protein, 9 of 10 DEGs for glutathione S-transferase, 8 of 10 DEGs for transcription factor MYB, 4 of 5 DEGs for calcineurin B-like protein, all 16 DEGs for receptor-like protein kinase, 15 of 17 DEGs for zinc finger $\mathrm{CCCH}$ domain-containing protein, all 14 DEGs for F-box protein, were up-regulated (Additional file 6). These up-regulated pathways and DEGs might also play important roles in drought tolerance in Eruca.

\section{Conclusions}

Based on the transcriptomic analyses we postulated that the most significantly up-regulated DEGs for Ethyleneresponsive transcription factors, WRKY and bHLH transcription factors involved in stress signaling and ABA responses; DEGs for calmodulin-binding transcription activator, cysteine-rich receptor-like protein kinase, mitogen-activated protein kinase kinase and WD repeatcontaining protein involved in antioxidants and ROS modulation; DEGs for OPDA reductase and allene oxide cyclase involved in JA production, C-5 sterol desaturase involved in producing D5, 7-sterols, aquaporin involved in decreasing ion leakage and malondialdehyde, Oacyltransferase WSD1 involved in cuticular wax biosynthesis; trehalose-phosphate phosphatase, galactinol synthase 4 and sugar transporter ERD 6-like 12 involved in osmoprotectant production, up-regulation of DEGs involved in alpha-linolenic acid metabolism, and down-regulation of carbon fixation and protein synthesis might be critical for the drought tolerance in Eruca line PI 426649. These results might be valuable for revealing mechanism of drought tolerance in Eruca and for improving drought tolerance in crops.

\section{Materials and methods Materials}

In this work Eruca vesicaria subsp. sativa PI 426649 highly tolerant and PI 426652 highly sensitive to PEGsimulated drought stress [19] were used for transcriptomic analysis.

\section{Methods}

Tissue sampling, RNA extraction, library preparation and Illumina sequencing

Eruca seeds of PI 426649 and PI 426652 originally from the Agricultural Research Service, USDA were germinated on filter paper immersed in liquid MS medium [87] without sugar or organic components. Seven days later the seedlings were treated for $10 \mathrm{~h}$ with $20 \%$ PEG6000 /liquid MS and then harvested and frozen imediately in liquid nitrogen and then stored at $-80{ }^{\circ} \mathrm{C}$. Drought-tolerant PI 426649 is denoted as 'DT and Drought-sensitive PI 426652 is denoted as 'DS'. Treatment with liquid MS medium is denoted as 'MS' while treatment with PEG is denoted as 'PEG'. Four samples (DT-MS, DT-PEG, DS-MS, DS-PEG) each with two biological replicates were taken for the present study. Total RNA was isolated from the whole seedlings that had been stored at $-80^{\circ} \mathrm{C}$ by using TRIZOL total RNA extraction reagent (TAKARA) according to the manufacturer's protocol. RNA integrity was verified by $1.5 \%$ Agrose gel electrophoresis and confirmed using a 2100 Bioanalyzer analyzer (Agilent, CA, USA). The mRNA enrichment, RNA fragmentation, the first and second strand cDNA synthesis and purfying, sequencing adaptors ligation and PCR amplification were performed as described [14]. The libraries were applied to Illumina sequencing platform (HiSeq 2000, SanDiego, CA, USA) for high-throughput sequencing using a paired-end read protocol with $100 \mathrm{bp}$ of data collected per run.

\section{De novo sequence assembly, clustering and homology search}

After sequencing, the raw image data was transformed into sequence data by base calling using CASAVA package provided by Illumina and saved as raw reads of fastq format and then treated with Trimmomatic(v0.30) to get clean reads. Transcriptome de novo assembly was carried out with short reads assembling program-Trinity (r2013-02-25) [88]. Unigenes from each sample's assembly were taken into further process of sequence splicing and redundancy removing to acquire non-redundant unigenes as long as possible by TGICL [89].

\section{Functional annotation}

Blastx alignment (E-value <0.00001) between unigenes and protein databases like $\mathrm{Nr}$ (NCBI non-redundant database), KEGG (Kyoto Encyclopedia Of Genes and Genomes, www.genome.jp/kegg/) and COG (Clusters of Orthologous Groups of proteins, http://www.ncbi.nlm. nih.gov/COG/) is performed as described [90]. After $\mathrm{Nr}$ annotation, Blast2GO program was used to get GO annotation for the unigenes. WEGO software was used to do GO functional classification for all unigenes and to understand the species distribution of gene functions at 
the macro level [91]. KEGG pathway annotation was carried out by using bidirectional best hit method $(\mathrm{BBH})$ at KEGG Automatic Annotation Server (KAAS).

\section{Analysis of differentially expressed genes (DEGs), GO and KEGG pathway enrichment}

Reads of the drought tolerant and sensitive Eruca accessions with PEG/MS treatment were mapped back to our de novo assembling results using RSEM [92]. To evaluate the gene expression, the number of unique match reads was calculated and then normalized to FPKM (Fragments per Kilo base of transcript per Million mapped reads) and then used to calculate the unigene expression with restrictive conditions of $\mid \log _{2}$ Ratio $\mid \geq 1.0$ and FDR $\leq 0.05$. GO enrichment analysis of these DEGs was performed using blast2GO with $P$-value $\leq 1$ and pathway enrichment analysis was carried out using Path finder software against the KEGG database with Q-value $\leq 1$.

Validation of the differentially expressed genes by $q R T-P C R$ To confirm the RNA-Seq result some transcript tags were selected for quantitative RT-PCR (qRT-PCR) analysis. The qRT-PCRs were performed in triplicate according to the Bioer (Hangzhou, China) manufacturer instructions and relative gene expression levels were determined by the $2^{-\Delta \Delta C T}$ method [93]. Primers listed in Additional file 9 for some DEGs were disgned and synthesized by GenScript (Nanjing) Co, Ltd. for qRT-PCR.

\section{Additional files}

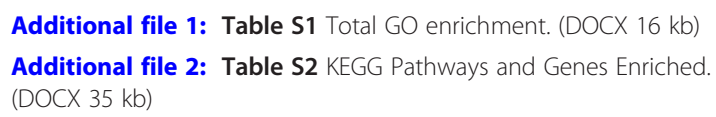

Additional file 3: Figure S1 DEGs in the DS-MS vs DS-PEG group enriched in 14 KEGG pathways. (DOCX $137 \mathrm{~kb}$ )

Additional file 4: Figure S2 DEGs in the DT-MS vs DT-PEG group enriched in 8 KEGG pathways. (DOCX $111 \mathrm{~kb}$ )

Additional file 5: Table S3 KEGG pathway enrichment for DEGs specifically in DT-MS vs DT-PEG. (DOCX 19 kb)

Additional file 6: Table S4 Differentially expressed unigenes specifically in DT-MS vs DT-PEG based on BLAST analysis. (DOCX 202 kb)

Additional file 7: Figure S3 COG enrichment for DEGs most significantly-up regulated specifically in DT-MS vs DT-PEG group. (DOCX $40 \mathrm{~kb}$ )

Additional file 8: Figure S4 KEGG pathway enrichment for DEGs most significantly-up regulated specifically in DT-MS vs DT-PEG group. (DOCX $58 \mathrm{~kb}$ )

Additional file 9: Table S5 Primers for quantitative RT-PCR. (DOCX 16 $\mathrm{kb})$

\section{Abbreviations}

ABA: Abscisic acid; AQPs: Aquaporins; BBH: Bidirectional best hit method; bHLH: Basic helix-loop-helix; CAMTA: Calmodulin-binding transcription activator; COG: Clusters of Orthologous Groups of proteins; CRKs: Cysteinerich receptor-like kinases; DEG: Differentially expressed gene; DS: Droughtsensitive; DT: Drought-tolerant; ERD: Early responsive to dehydration; ERF: Ethylene-responsive transcription factors; FDR: False Discovery Rate;
FPKM: Fragments per Kilo base of transcript per Million mapped reads; GO: Gene ontology; GolS: Galactinol synthase; IL: Ion leakage; JA: Jasmonic acid; KAAS: KEGG Automatic Annotation Server; KEGG: Kyoto Encyclopedia Of Genes and Genomes; MDA: Malondialdehyde; MS: Murashige-Skoog; NGS: Next generation sequencing; Nr: NCBI non-redundant database; OPDA: 12-oxophytodienoate; PEG: Polyethylene glycol; q-RT-

PCR: Quantitative RT-PCR; ROS: Reactive oxygen species

\section{Acknowledgements}

Thanks to GENEWIZ at Suzhou, China, for helping with the transcriptional analysis

\section{Authors' contributions}

$H B, L Z$ and $Z X$ conceived the experiments and finalized the paper, $H B-L$ performed most of the experiments and prepared the draft manuscript, LX, LP, ML and WW helped with material preparation, RNA isolation, GRT-PCR and data analyses. All authors read and approved the final manuscript.

\section{Funding}

Material collection and drought tolerance evaluation in this study were financially supported by the National Key Research and Development Program of China (2016YFD0100202-23; 2017YFD0101701); transcriptomic sequencing and data analyses were supported by the National Natural Science Foundation of China (31771829).

\section{Availability of data and materials}

The datasets generated and/or analysed in this study are available from the corresponding author on reasonable request.

Ethics approval and consent to participate Not Applicable.

Consent for publication

Not applicable.

\section{Competing interests}

The authors declare that they have no competing interests.

\section{Author details}

${ }^{1}$ Hubei Collaborative Innovation Center for Green Transformation of Bio-Resources, College of Life Science, Hubei University, Wuhan 430062, China. ${ }^{2}$ Jiangsu Microbe Biological \& Environmental Engineering Co., Ltd, Wuxi 214122, China. ${ }^{3}$ Key Laboratory of Biology and Genetic Improvement of Oil Crops, Ministry of Agriculture, Oil Crops Research Institute of the Chinese Academy of Agricultural Sciences, Wuhan 430062, China. ${ }^{4}$ National Key Lab of Crop Genetic Improvement, College of Plant Science and Technology, Huazhong Agricultural University, Wuhan 430070, China.

Received: 22 February 2018 Accepted: 29 August 2019

Published online: 11 October 2019

\section{References}

1. Boyer JS. Plant productivity and environment. Science. 1982;218:443-8.

2. Mittler R. Abiotic stress, the field environment and stress combination. Trends Plant Sci. 2006;11:15-9.

3. Mittler R, Blumwald E. Genetic engineering for modern agriculture: challenges and perspectives. Annu Rev Plant Biol. 2010;61:443-62.

4. Price $\mathrm{AH}$, Cairns JE, Horton $\mathrm{P}$, Jones $\mathrm{HG}$, Griffiths $\mathrm{H}$. Linking droughtresistance mechanisms to drought avoidance in upland rice using a QTL approach: progress and new opportunities to integrate stomatal and mesophyll responses. J Exp Bot. 2002;53:989-1004.

5. Shinozaki K, Yamaguchi-Shinozaki K, Seki M. Regulatory network of gene expression in the drought and cold stress responses. Curr Opin Plant Biol. 2003;6(5):410-7.

6. Shinozaki K, Yamaguchi-Shinozaki K. Gene networks involved in drought stress response and tolerance. J Exp Bot. 2007;58:221-7.

7. Hao Z, Li X, Liu X, Xie C, Li M, Zhang D, et al. Meta-analysis of constitutive and adaptive QTL for drought tolerance in maize. Euphytica. 2010;174(2):165-77.

8. Morozova O, Marra MA. Applications of next-generation sequencing technologies in functional genomics. Genomics. 2008;92(5):255-64. 
9. Shendure J, Ji H. Next-generation DNA sequencing. Nat Biotechnol. 2008;26:1135-45.

10. Mortazavi A, Williams BA, McCue K, Schaeffer L, Wold B. Mapping and quantifying mammalian transcriptomes by RNA-Seq. Nat Meth. 2008:5:621-8.

11. Stolf-Moreira R, Lemos EGM, Carareto-Alves L, Marcondes J, Pereira SS, Rolla AAP, et al. Transcriptional profiles of roots of different soybean genotypes subjected to drought stress. Plant Mol Biol Rep. 2011;29:19-34.

12. Lenka SK, Katiyar A, Chinnusamy V, Bansal KC. Comparative analysis of drought-responsive transcriptome in Indica rice genotypes with contrasting drought tolerance. Plant Biotechnol J. 2011:9:315-27.

13. Chen LM, Zhou XA, Li WB, Chang W, Zhou R, Wang C, et al. Genome-wide transcriptional analysis of two soybean genotypes under dehydration and rehydration conditions. BMC Genomics. 2013;14:687.

14. Xu J, Yuan $Y, X u$ Y, Zhang G, Guo X, Wu F, et al. Identification of candidate genes for drought tolerance by whole-genome resequencing in maize. BMC Plant Biol. 2014;14:83.

15. Tian X-J, Long Y, Wang J, Zhang J-W, Wang Y-Y, Li W-M, et al. De novo Transcriptome Assembly of Common Wild Rice (Oryza rufipogon Griff.) and Discovery of Drought-Response Genes in Root Tissue Based on Transcriptomic Data. PloS ONE. 2015;10(7):e0131455.

16. Barrios A, Caminero C, García P, Krezdorn N, Hoffmeier K, Winter P, de la Vega MP. Deep super-SAGE transcriptomic analysis of cold acclimation in lentil (Lens culinaris Medik.). BMC Plant Biol. 2017;17:111.

17. Sun W, Yang Q, Zhang J, Zhang T, Yun Z. Assessment on drought tolerance of Eruca sativa genotypes from northwestern China. Proc GCIRC 10th Int. Rapeseed Congr. 1999; 4: 628.

18. Prakash S, Bhat SR. Contribution of wild crucifers in Brassica improvement: past accomplishment and future perspectives. Proc. GCIRC 12th Int. Rapeseed Congr 2007; 1: 213-215.

19. Huang B, Su J, Zhang G, Luo X, Wang H, Gao Y, et al. Screening for Eruca genotypes tolerant to polyethylene glycol-simulated drought stress based on the principal component and cluster analyses of seed germination and early seedling growth. Plant Genetic Resour. 2017;15(2):187-93.

20. Audic S, Claverie JM. The significance of digital gene expression profiles. Genome Res. 1997;7:986-95.

21. Hayano-Kanashiro C, Calderon-Vazquez C, Ibarra-Laclette E, Herrera-Estrella L, Simpson J. Analysis of gene expression and physiological responses in three Mexican maize landraces under drought stress and recovery irrigation. PLoS One. 2009:4:e7531.

22. Cutler SR, Rodriguez PL, Finkelstein RR, Abrams SR. Abscisic acid: emergence of a core signaling network. Annu Rev Plant Biol. 2010;61:651-79.

23. Raghavendra AS, Gonugunta VK, Christmann A, Grill E. ABA perception and signalling. Trends Plant Sci. 2010;15:395-401.

24. Weiner JJ, Peterson FC, Volkman BF, Cutler SR. Structural and functional insights into core ABA signaling. Curr Opin Plant Biol. 2010;13:495-502.

25. Rushton DL, Tripathi P, Rabara RC, Lin J, Ringler P, Boken AK, et al. WRKY transcription factors: key components in abscisic acid signaling. Plant Biotechnol J. 2012;10:2-11.

26. Qiu Y, Yu D. Over-expression of the stress-induced OsWRKY45 enhances disease resistance and drought tolerance in Arabidopsis. Environ Exp Bot. 2009;65(1):35-47.

27. Jiang S, Kumar S, Eu YJ, Jami SK, Stasolla C, Hill RD. The Arabidopsis mutant, fy1, has an ABA-insensitive germination phenotype. J Exp Bot. 2012;63:2693-703.

28. Seo JS, Joo J, Kim MJ, Kim YK, Nahm BH, Song SI, et al. OsbHLH148, a basic helix-loop-helix protein, interacts with OsJAZ proteins in a jasmonate signaling pathway leading to drought tolerance in rice. Plant J. 2011;65(6):907-21.

29. Liu W, Tai H, Li S, Gao W, Zhao M, Xie C, et al. bHLH122 is important for drought and osmotic stress resistance in Arabidopsis and in the repression of ABA catabolism. New Phytol. 2013;2:1-7.

30. Dong Y, Wang C, Han X, Tang S, Liu S, Xia X, et al. A novel bHLH transcription factor PebHLH35 from Populus euphratica confers drought tolerance through regulating stomatal development, photosynthesis and growth in Arabidopsis. Biochem Biophys Res Commu. 2014;450:453-8.

31. Schmidt R, Mieulet D, Hubberten HM, Obata T, Hoefgen R, Fernie AR, et al. SALTRESPONSIVE ERF1 regulates reactive oxygen species-dependent signaling during the initial response to salt stress in rice. Plant Cell. 2013;25(6):2115-31.

32. Jin $L G$, Liu JY. Molecular cloning, expression profile and promoter analysis of a novel ethylene responsive transcription factor gene GhERF4 from cotton (Gossypium hirsutum). Plant Physiol Bioch. 2008;46:46-53.

33. Tang M, Sun J, Liu Y, Chen F, Shen S. Isolation and functional characterization of the JCERF gene, a putative AP2/EREBP domain- containing transcription factor, in the woody oil plant Jatropha curcas. Plant Mol Biol. 2007:63:419-28.

34. Quan R, Hu S, Zhang Z, Zhang H, Zhang Z, Huang R. Overexpression of an ERF transcription factor TSRF1 improves rice drought tolerance. Plant Biotechnol J. 2010;8(4):476-88.

35. Zhang C, Zhang L, Zhang S, Zhu S, Wu P, Chen Y, et al. Global analysis of gene expression profiles in physic nut (Jatropha curcas L.) seedlings exposed to drought stress. BMC Plant Biol. 2015;15:17.

36. Rong W, Qi L, Wang A, Ye X, Du L, Liang H, et al. The ERF transcription factor TaERF3 promotes tolerance to salt and drought stresses in wheat. Plant Biotechnol J. 2014;12(4):468-79.

37. Hubbard KE, Nishimura N, Hitomi K, Getzoff ED, Schroeder Jl. Early abscisic acid signal transduction mechanisms: newly discovered components and newly emerging questions. Genes Dev. 2010;24:1695-708.

38. Pandey N, Ranjan A, Pant P, Tripathi RK, Ateek F, Pandey HP, et al. CAMTA 1 regulates drought responses in Arabidopsis thaliana. BMC Genomics. 2013;14:216.

39. Wrzaczek M, Brosche M, Salojarvi J, Kangasjarvi S, Idanheimo N, Mersmann $\mathrm{S}$, et al. Transcriptional regulation of the CRK/DUF26 group of receptor-like protein kinases by ozone and plant hormones in Arabidopsis. BMC Plant Biol. 2010;10:95.

40. Zhang X, Yang G, Shi R, Han X, Qi L, Wang R, et al. Arabidopsis cysteine-rich receptor-like kinase 45 functions in the responses to abscisic acid and abiotic stresses. Plant Physiol Biochem. 2013;67:189-98.

41. Lu W, Chu X, Li Y, Wang C, Guo X. Cotton GhMKK1 induces the tolerance of salt and drought stress, and mediates defence responses to pathogen infection in transgenic Nicotiana benthamiana. PLoS One. 2013;8:e68503.

42. Tena G, Asai T, Chiu WL, Sheen J. Plant mitogen-activated protein kinase signaling cascades. Curr Opin Plant Biol. 2001;4:392-400.

43. Jonak $C$. ökrész L, Bögre L, Hirt H. complexity, cross talk and integration of plant MAP kinase signaling. Curr Opin Plant Biol. 2002;5:415-24.

44. Mittler R. Oxidative stress, antioxidants and stress tolerance. Trends Plant Sci. 2002:7:405-10.

45. Samuel MA, Ellis BE. Double jeopardy: both overexpression and suppression of a redox-activated plant mitogen-activated protein kinase render tobacco plants ozone sensitive. Plant Cell. 2002;14:2059-69.

46. Moon H, Lee B, Choi G, Shin D, Prasad DT, Lee O, et al. NDP kinase 2 interacts with two oxidative stress-activated MAPKs to regulate cellular redox state and enhances multiple stress tolerance in transgenic plants. Proc Natl Acad Sci U S A. 2003;100:358-63.

47. Neill SJ, Desikan R, Clarke A, Hurst RD, Hancock JT. Hydrogen peroxide and nitric oxide as signaling molecules in plants. J Exp Bot. 2002;53:1237-47.

48. Mizoguchi T, Ichimura K, Irie K, Morris P, Giraudat J, Matsumoto K, et al. Identification of a possible MAP kinase cascade in Arabidopsis thaliana based on pairwise yeast two hybrid analysis and functional complementation tests of yeast mutants. FEBS Lett. 1998;437:56-60.

49. Mikołajczyk M, Awotunde OS, Muszyńska G, Klessig DF, Dobrowolska G. Osmotic stress induces rapid activation of a salicylic acid-induced protein kinase and a homolog of protein kinase ASK1 in tobacco cells. Plant Cell. 2000;12:165-78.

50. Xing Y, Jia W, Zhang J. AtMKK1 mediates ABA-induced CAT1 expression and $\mathrm{H}_{2} \mathrm{O}_{2}$ production via AtMPK6-coupled signaling in Arabidopsis. Plant J. 2008:54:440-51.

51. Kim ED, Chen ZJ. Unstable transcripts in Arabidopsis allotetraploids are associated with nonadditive gene expression in response to abiotic and biotic stresses. PLoS One. 2011;6:e24251.

52. Fang $Y$, Liao $K$, Du H, Xu Y, Song H, Li X, Xiong LA. Stress-responsive NAC transcription factor SNAC3 confers heat and drought tolerance through modulation of reactive oxygen species in rice. J Exp Bot. 2015;25:3472-90.

53. Upchurch RG. Fatty acid unsaturation, mobilization, and regulation in the response of plants to stress. Biotechnol Lett. 2008;30:967-77.

54. Demmig-Adams B, Cohu CM, Amiard V, van Zadelhoff G, Veldink GA, et al. Emerging trade-offs - impact of photoprotectants (PsbS, xanthophylls, and vitamin E) on oxylipins as regulators of development and defense. New Phytol. 2013;197:720-9.

55. Gfeller A, Dubugnon L, Liechti R, Farmer EE. Jasmonate biochemical pathway. Sci Signal. 2010;3:3.

56. Staswick PE. JAZing up jasmonate signaling. Trends Plant Sci. 2008;13:66-71.

57. Im YJ, Han O, Chung GC, Cho BH. Antisense expression of an Arabidopsis omega-3 fatty acid desaturase gene reduces salt / drought tolerance in transgenic tobacco plants. Mol Cells. 2002;13:264-71.

58. Zhang M, Barg R, Yin M, Gueta-Dahan Y, Leikin-Frenkel A, Salts Y, et al. Modulated fatty acid desaturation via overexpression of two distinct 
omega-3 desaturases differentially alters tolerance to various abiotic stresses in transgenic tobacco cells and plants. Plant J. 2005;44:361-71.

59. Creelman RA, Mullet JE. Jasmonic acid distribution and action in plants: regulation during development and response to biotic and abiotic stress. Proc Natl Acad Sci U S A. 1995;92:4114-9.

60. Brossa R, Lopez-Carbonell M, Jubany-Mari T, Alegre L. Interplay between abscisic acid and Jasmonic acid and its role in water-oxidative stress in wildtype, ABA-deficient, JA-deficient, and ascorbate-deficient Arabidopsis plants. J Plant Growth Regul. 2011;30:322-33.

61. Vick BA, Zimmerman DC. Biosynthesis of jasmonic acid by several plant species. Plant Physiol. 1984;75:458-61.

62. Simpson TD, Gardner HW. Allene oxide synthase and allene oxide cyclase, enzymes of the jasmonic acid pathway, localized in Glycine max tissues. Plant Physiol. 1995;108:199-202.

63. Maucher H, Hause B, Feussner I, Ziegler J, Wasternack C. Allene oxide synthases of barley (Hordeum vulgare cv. Salome): tissue specific regulation in seedling development. Plant J. 2000;21:199-213.

64. Leon J, Royo J, Vancanneyt G, Sanz C, Silkowski H, GriYths G, SanchezSerrano JJ. Lipoxygenase $\mathrm{H} 1$ gene silencing reveals a specific role in supplying fatty acid hydroperoxides for aliphatic aldehyde production. J Biol Chem. 2002;277:416-23.

65. Koo AJK, Chung HS, Kobayashi Y, Howe GA. Identification of a peroxisomal acyl-activating enzyme involved in the biosynthesis of jasmonic acid in Arabidopsis. J Biol Chem. 2006;281:33511-20.

66. Kienow L, Schneider K, Bartsch M, Stuible HP, Weng H, Miersch O, et al. Jasmonates meet fatty acids: functional analysis of a new acyl-coenzyme a synthetase family from Arabidopsis thaliana. J Exp Bot. 2008;59:403-19.

67. Montillet JL, Chamnongpol S, Rusterucci C, Dat J, Cotte B, Agnel JP, et al. Fatty acid hydroperoxides and $\mathrm{H}_{2} \mathrm{O}_{2}$ in the execution of hypersensitive cell death in tobacco leaves. Plant Physiol. 2005;138:1516-26.

68. Xu Y, Hu W, Liu J, Zhang J, Jia C. Miao H, et al a banana aquaporin gene, MaPIP1;1, is involved in tolerance to drought and salt stresses. BMC Plant Biol. 2014;14:59.

69. Riederer M, Schreiber L. Protecting against water loss: analysis of the barrier properties of plant cuticles. J Exp Bot. 2001;52(363):2023-32.

70. Kamthan A, Kamthan M, Azam M, Chakraborty N, Chakraborty S, Datta A Expression of a fungal sterol desaturase improves tomato drought tolerance, pathogen resistance and nutritional quality. Sci Rep. 2012;2:951.

71. Li F, Wu X, Lam P, Bird D, Zheng H, Samuels $L$, et al. Identification of the wax ester synthase/acyl-coenzyme a: diacylglycerol acyltransferase WSD1 required for stem wax ester biosynthesis in Arabidopsis. Plant Physiol. 2008;148(1):97-107.

72. Wormit A, Trentmann O, Feifer I, Lohr C, Tjaden J, Meyer S, et al. Molecular identification and physiological characterization of a novel monosaccharide transporter from Arabidopsis involved in vacuolar sugar transport. Plant Cell. 2006;18:3476-90.

73. Schulze W. Proteomics approaches to understand protein phosphorylation in pathway modulation. Curr Opin Plant Biol. 2010;13:1-8.

74. Rizhsky L, Liang H, Shuman J, Shulaev V, Davletova S, Mittler R. When defense pathways collide. The response of Arabidopsis to a combination of drought and heat stress. Plant Physiol. 2004;134:1683-96.

75. Kiyosue T, Abe H, Yamaguchi-Shinozaki K, Shinozaki K. ERD6, a cDNA clone for an early dehydration-induced gene of Arabidopsis, encodes a putative sugar transporter. Biochim Biophys Acta. 1998;1370:187-91.

76. Yamada K, Osakabe Y, Mizoi J, Nakashima K, Fujita Y, Shinozaki K, et al. Functional analysis of an Arabidopsis thaliana abiotic stress-inducible facilitated diffusion transporter for monosaccharides. J Biol Chem. 2010;285:1138-46.

77. Elbein AD, Pan YT, Pastuszak I, Carroll D. New insights on trehalose: a multifunctional molecule. Glycobiology. 2003;13:17R-27R.

78. Lunn JE, Feil R, Hendriks JH, Gibon Y, Morcuende R, Osuna D, et al. Sugarinduced increases in trehalose 6-phosphate are correlated with redox activation of ADPglucose pyrophosphorylase and higher rates of starch synthesis in Arabidopsis thaliana. Biochem J. 2006;397:139-48.

79. Paul MJ, Primavesi LF, Jhurreea D, Zhang YH. Trehalose metabolism and signaling. Annu Rev Plant Bio. 2008;59:417-41.

80. Saravitz DM, Pharr DM, Carter TE. Galactinol synthase activity and soluble sugars in developing seeds of four soybean genotypes. Plant Physiol. 1987;83:185-9.

81. Ingram J, Bartels D. The molecular basis of dehydration tolerance in plants. Annu. Rev Plant Physiol Plant Mol Biol. 1996:47:377-403.

82. Bray EA. Plant responses to water deficit. Trends Plant Sci. 1997;2:48-54.

83. Shinozaki K, Yamaguchi-Shinozaki K. Molecular responses to drought and cold stress. Curr Opin Biotechnol. 1997;7:161-7.
84. Taji T, Ohsumi C, luchi S, Seki M, Kasuga M, Kobayashi M, et al. Important roles of drought- and cold-inducible genes for galactinol synthase in stress tolerance in Arabidopsis thaliana. Plant J. 2002;29:417-26.

85. Himuro Y, Ishiyama K, Mori F, Gondo T, Takahashi F, Shinozaki K, et al. Arabidopsis galactinol synthase AtGolS2 improves drought tolerance in the monocot model Brachypodium distachyon. J Plant Physiol. 2014;171:1127-31.

86. Dhindsa RS, Bewley JD. Water stress and protein synthesis. IV. Responses of a drought-tolerant plant. J Exp Bot. 1976;27(98):513-23.

87. Murashige T, Skoog F. A revised medium for rapid growth and bio assays with tobacco tissue cultures. Physiol Plant. 1962;15(3):473-97.

88. Haas BJ, Papanicolaou A, Yassour M, Grabherr M, Blood PD. De novo transcript sequence reconstruction from RNA-seq using the trinity platform for reference generation and analysis. Nat Protoc. 2013;8:1494-512.

89. Pertea G, Huang X, Liang F, Antonescu V, Sultana R, Karamycheva S, et al. TIGR gene indices clustering tools (TGICL): a software system for fast clustering of large EST datasets. Bioinformatics. 2003;19:651-2.

90. Zhu G, Wang L, Tang W, Liu D, Yang J. De novo transcriptomes of olfactory epithelium reveal the genes and pathways for spawning migration in japanese grenadier anchovy (Coilia nasus). PLoS One. 2014;9(8):e103832.

91. Ye J, Fang L, Zheng H, Zhang Y, Chen J, Zhang Z, et al. WEGO: a web tool for plotting GO annotations. Nucleic Acids Res (Web Server issue). 2006;34:W293-7.

92. Li B, Dewey CN. RSEM: accurate transcript quantification from RNA-Seq data with or without a reference genome. BMC Bioinformatics. 2011;12:323.

93. Livak KJ, Schmittgen TD. Analysis of relative gene expression data using real-time quantitative PCR and the $2^{\Delta \Delta C}$ T method. Methods. 2001;25:402-8.

\section{Publisher's Note}

Springer Nature remains neutral with regard to jurisdictional claims in published maps and institutional affiliations.

\section{Ready to submit your research? Choose BMC and benefit from:}

- fast, convenient online submission

- thorough peer review by experienced researchers in your field

- rapid publication on acceptance

- support for research data, including large and complex data types

- gold Open Access which fosters wider collaboration and increased citations

- maximum visibility for your research: over $100 \mathrm{M}$ website views per year

At BMC, research is always in progress.

Learn more biomedcentral.com/submissions 